\title{
Determination of structural homogeneity of synthetic diamonds from analysis of Kikuchi lines intensity distribution
}

\author{
I. Fodchuk ${ }^{1,2}$, S. Balovsyak ${ }^{1,2}$, M. Borcha ${ }^{1,2}$, Ya. Garabazhiv ${ }^{1}$, V. Tkach $^{3}$ \\ ${ }^{I} Y u$. Fedkovych Chernivtsi National University, 2, Kotsyubinskiy str, 58012 Chernivtsi, Ukraine \\ ${ }^{2}$ Institute for Metal Physics, NAS of Ukraine, 36, Acad. Vernadskiy blvd., 03142 Kyiv, Ukraine \\ ${ }^{3}$ V.N. Bakul Institute of Superhard Materials, NAS of Ukraine, 2, Avtozavodska str., 03142 Kyiv, Ukraine
}

\begin{abstract}
It has been suggested the technique based on analysis of geometry and intensity distribution profiles in Kikuchi patterns obtained due to electron backscattering diffraction for defining structural imperfection of diamond crystals. To determine the geometry parameters in Kikuchi patterns with the maximal precision, the special software was developed. It has been shown that application of electron diffraction (Kikuchi method) allows to obtain information about degree of perfection and homogeneity of real structure for diamond crystals synthesized with various methods.
\end{abstract}

Keywords: electron diffraction, Kikuchi line profile, synthesized diamond, local strains, structural imperfection.

Manuscript received 14.01.10; accepted for publication 08.07.10; published online 30.09.10.

\section{Introduction}

Investigation of physical and mechanical properties of diamond single crystals and films is accompanied by a number of difficulties due to the small sizes of diamonds and their complex zonal-sectorial structure caused by selective capture of impurities during the growth of different crystal faces [1-10]. Determination of the elastic characteristics of diamond is even more acute problem [9]. For a film of polycrystalline materials, the orientation (texture) of crystallites and their sizes are significant object for study [13].

The electron backscattering diffraction (Kikuchi's method) is very promising to solve these problems [1217]. An opportunity to obtain electron backscattering diffraction patterns for the local crystal regions with size of $\sim 20 \mathrm{~nm}$ takes place by using the Kikuchi method. It is extremely important in the study of samples with inhomogeneous structure. This method allows determining the changes in structural parameters (lattice parameters, inhomogeneous distribution of impurities in local areas of grains, etc.) with a high accuracy $(\sim 0.01 \%)$ [12-13], and is very convenient for determining the angular orientation of individual grains in polycrystalline samples. In addition, the transformation of the fine structure in the Kikuchi line profile gives opportunity to obtain additional information about the type and distribution of local structural defects in the bulk during the process of crystal growth [12-16]. It is applied particularly to determine the type of defects and the nature of strain field changes from the analysis of the intensity distribution features in the cross-sections of Kikuchi lines.

In this paper, we propose the method to analyze the structural perfection of diamond crystals, which is based on the analysis of geometry and intensity profiles in Kikuchi lines. Specialized software was created for the most accurate determination of geometric parameters in Kikuchi patterns.

\section{Experimental}

Using a scanning electron microscope made by "Zeiss" EVO-1950 using CCD-detector, Kikuchi patterns were obtained (Figs 1 to 4) for a series of samples of synthesized diamonds $(\mathrm{N} 1-\mathrm{N} 4)$. The electron beam was incident on the crystal surface at the angle of $70^{\circ}$.

Objects of research: diamonds N1, N3 and N4 were obtained by spontaneous method in growth systems $\mathrm{Fe}$ $\mathrm{Co}-\mathrm{C}$ and Ni-Mn-C at a high static pressure and high temperature in the presence of metal catalysts (solvent) $\mathrm{Fe}-\mathrm{Co}$ and Ni-Mn, respectively. These growth systems are characterized by the following conditions: pressure $\sim 4.5 \ldots 6 \mathrm{GPa}$ and temperature close to $\sim 1650 \mathrm{~K}$. Crystals were characterized by the zonal-sectorial structure, which is due to the uneven distribution of nitrogen and boron impurities during crystal growth, and selective entry into the pyramid of growth in the $\langle 100\rangle$ and $\langle 111\rangle[6-11]$. 

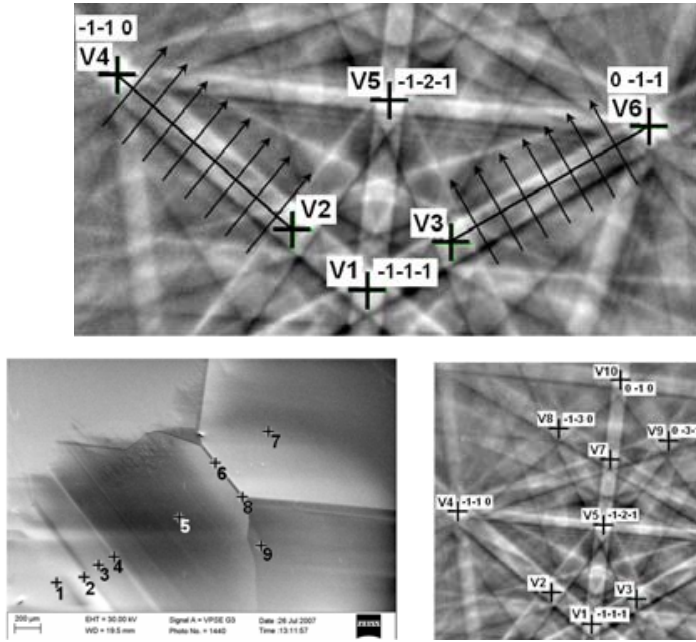

a)

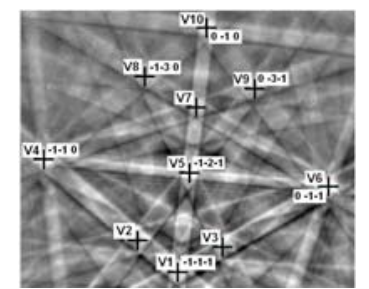

b)

Fig. 1. Crystal of synthesized diamond N1: a) cathodoluminescence image in secondary electrons for $(00 \overline{1})$ surface fragment of $3.0 \times 2.0 \mathrm{~mm}$; numbers indicate areas from which Kikuchi patterns were obtained; b) Kikuchi pattern of area 1, markers "+" indicate sites V1-V10 of Kikuchi lines intersections.

The diamond N2 was obtained using the temperature gradient method at the pressure $\sim 5.7 \mathrm{GPa}$ and temperature $1600 \mathrm{~K}$. The crystal was obtained from a "seed" (diamond plate) and polished parallel to the $\langle 100\rangle$. The growth rate of these diamonds was usually $\sim 2.7 \mathrm{mg}$ /hour, which contributes to the minimum entrainment of the catalyst crystal inclusions during growth and reduces the lattice strains [6].

The Kikuchi patterns obtained with backscattering electrons are represented in Figs $1 \mathrm{~b}$ to $4 \mathrm{~b}$. There is a system of intersecting bands called Kikuchi lines. Since in the case of diffraction of fast electrons, diffraction angles $\theta$ are sufficiently small, the intersection of cones of reflected electrons and the detector plane has the form of straight lines (instead of hyperbole at the X-ray diffraction). These patterns were obtained from local crystal areas, which have differences in structure and are marked by numbers in Figs 1a to $4 \mathrm{a}$.

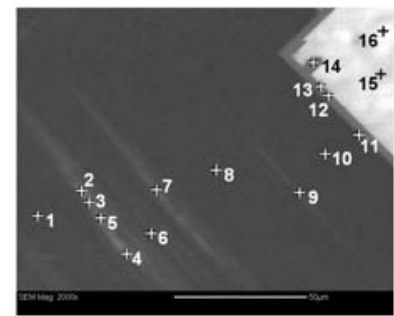

a)

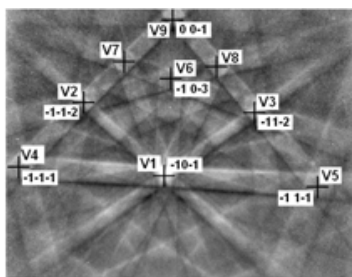

b)
Fig. 2. Crystal of synthesized diamond N2: a) cathodoluminescence image in secondary electrons for $(0 \overline{1} 0)$ surface fragment of $140 \times 100 \mu \mathrm{m}$; numbers indicate areas from which Kikuchi patterns were obtained; bright rectangular right top area is the single-crystal seed; b) Kikuchi pattern of the area 1.

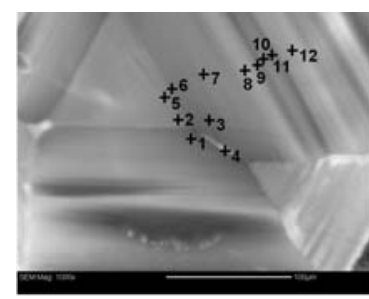

a)

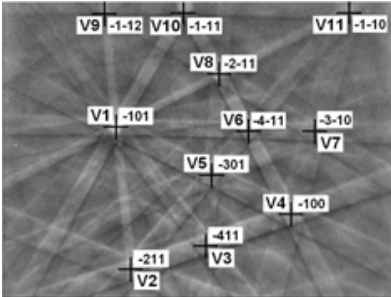

b)
Fig. 3. Crystal of synthesized diamond N3: a) cathodoluminescence image in secondary electrons for ( $\overline{1} 11)$ surface fragment of $280 \times 200 \mu \mathrm{m}$; numbers indicate areas from which Kikuchi patterns were obtained; b) Kikuchi pattern of the area 1 .

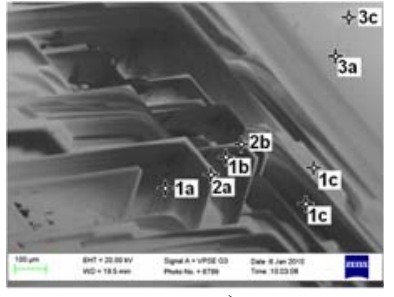

a)

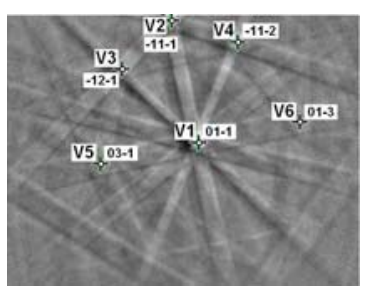

b)
Fig. 4. Crystal of synthesized diamond N4: a) cathodoluminescence image in secondary electrons for ( $\overline{1} 11)$ surface fragment of $1.2 \times 0.8 \mathrm{~mm}$; numbers indicate areas from which Kikuchi patterns were obtained; b) Kikuchi pattern of the area 1a.

In general, the patterns of Kikuchi lines are very sensitive to changes in crystal orientation [14]. The accuracy of determining the lattice parameter depends on the accuracy of geometrical parameters, such as coordinates of poles (sites) that are regions of intersection of the diffraction lines in the Kikuchi patterns [12]. In spite of this, the Kikuchi patterns differ one from another by small displacements of sites, and by their width, i.e. distances between maxima and minima of the intensity profile.

The analysis of intensity distributions for the diffraction lines in the Kikuchi patterns (Figs 1 to 4), obtained in the areas identified by numbers on the cathode-luminescence images of crystal surfaces was made to determine local changes in the lattice parameter.

\section{Determination of the distribution profiles for the Kikuchi lines intensity}

The trace of reflected crystal plane, received during electron diffraction, accurately projects in the middle between the light and dark Kikuchi lines (minimum and maximum) and is a gnomonic projection of the reflecting planes $(h k l),(\bar{h} \bar{k} \bar{l})$ (Fig. 5) [16]. The distance $L$ between the Kikuchi lines in the angular scale corresponds to the angle $2 \theta$, and in the linear scale is given by [14] 
$L=L_{W} \cdot 2 \cdot \theta=L_{W} \cdot n \lambda / d_{h k l}=c / d_{h k l}$,

where $L_{W}$ is the effective distance between object and screen, $c$ is the electron diffraction constant; $d_{h k l}$ is the distance between planes.

The distortion of Kikuchi patterns arising from differences in geometry of the experiment can be removed by the corresponding scaling of images, correcting brightness and contrast [18-21]. Relative changes in intensity profiles for individual lines are insignificant, but with the suppression of noise by averaging the intensity of profiles in Fig. 5 a characteristic shape of the profile exhibits, which also depends on the direction of the line and the local structural perfection of the investigated region in the crystal.
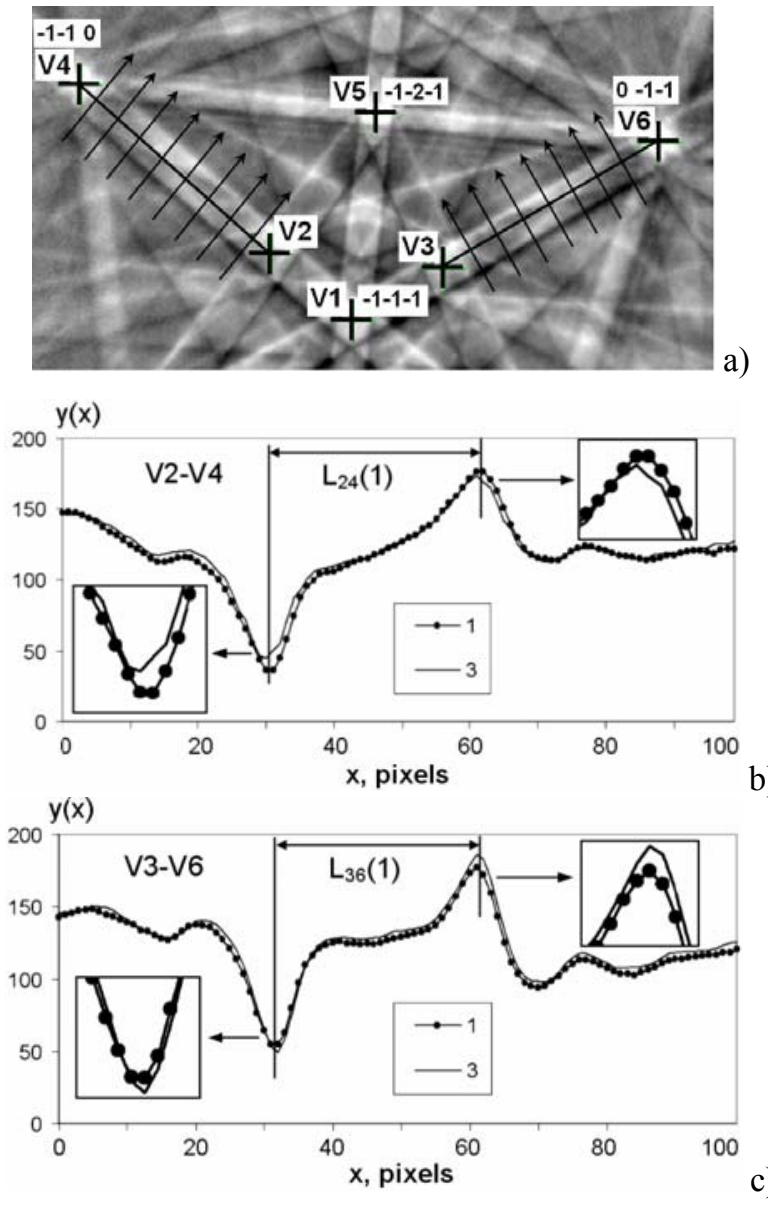

b)

Fig. 5. Crystal N1: a) cathodoluminescence image; b) and c) profiles of intensity distribution for $(00 \overline{1})$ and $(\overline{1} 00)$ Kikuchi lines, respectively; curve with circles corresponds to the area 1 , curve without circles corresponds to the area 3 ; $(00 \overline{1})$ line width between V2-V4 sites is equal to $L_{24}(1)=30.59$ pixels for the area 1 and $L_{24}(3)=30.43$ pixels for the area $3 ;(\overline{1} 00)$ line width between V3-V6 sites is equal to $L_{36}(3)=27.75$ pixels for the area 1 and $L_{36}(3)=27.87$ pixels for the area 3 .
The intensity distribution for certain Kikuchi lines in Fig. 5 was determined using a specially developed algorithm and software "Image Profile" created in Delphi. The program "Image_Profile" allows one to read the individual profiles and average a series of profiles along a certain direction. Due to averaging, the random component intensity (noise) is decreased [19]. Incidentally, the difference between the intensity distributions of the individual sections of the lines in Fig. 5 indicates the anisotropy of the structure of these samples in different crystallographic directions.

To determine the distance between the extremes of the Kikuchi lines (minimum and maximum, light and dark stripes), a specialized program "Fit_Kikuchi" was created, the basis of which has the following algorithm: the initial profile (one minimum and one maximum $-m_{i}$ ) is read from the file, which can then be approximated by Gaussian distribution $\left(m_{i f}\right)$. In the process of fitting $m_{i}$ to the $m_{i f}$, the coordinates of the center $X_{C}$ and standard deviation $\sigma$ of Gaussian distribution are varied by minimizing the value of the standard deviation difference

$C_{r}=\sqrt{\sum_{i=1}^{n}\left(m_{i}-m_{i f}\right)^{2}}$,

where $n$ is the number of profile points.

In the process of fitting the profiles difference $C_{r}$ is calculated only in the range $\left(X_{C}-\sigma, X_{C}+\sigma\right)$ relatively to the center $X_{C}$ of Gaussian distribution, which let to determine the width of the Kikuchi lines more accurately (Fig. 6). Using the "Fit_Kikuchi", the dependence of the line width $L$ (distance between peaks) in the number of areas (Figs 6 and 7) for diamonds N1-N4 was determined.
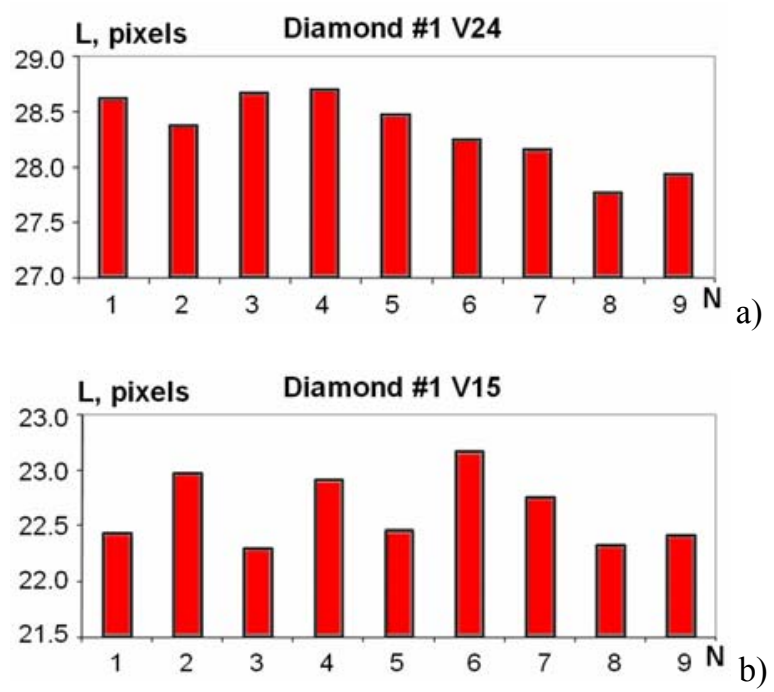

Fig. 6. Values of the Kikuchi line width for the crystal N1: a) V24 is $(00 \overline{1})$ line width between V2 and V4 sites; b) V15 is $(\overline{1} 00)$ line width between $\mathrm{V} 1$ and $\mathrm{V} 5$ sites. $\mathrm{N}$ is the number of a crystal area. 

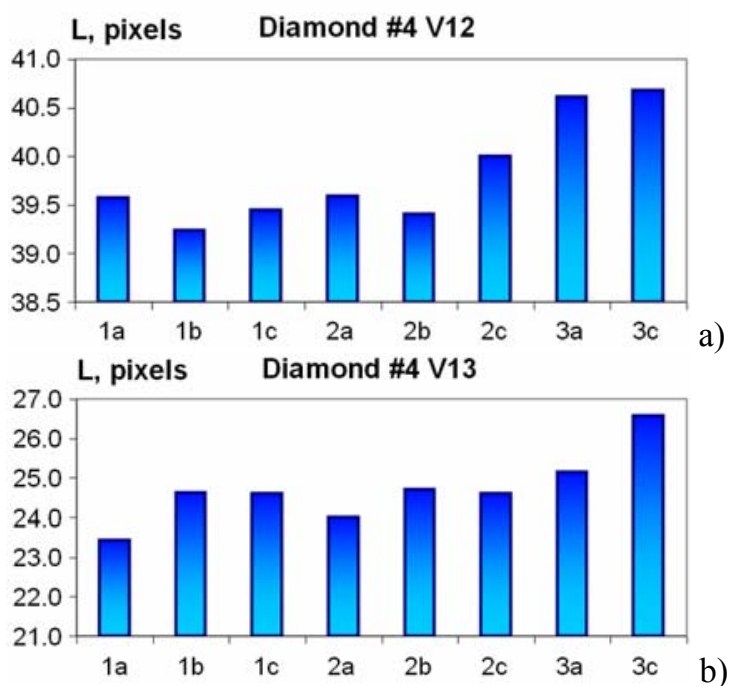

Fig. 7. Values of the Kikuchi line width for the crystal N4: a) V12 is (100) line width between V1 and V2 sites; b) V13 is (1 $1 \overline{1} 0)$ line width between $\mathrm{V} 1$ and V3 sites. $1 \mathrm{a}-3 \mathrm{c}$ are the numbers of a crystal area.

\section{Determination of changes of interplanar spacing in the local regions of crystals}

The analysis of changes in the line width $L$ permits to specify relative changes in the interplanar spacing $\Delta d / d$ in the local regions of diamond crystals N1-N4 (Fig. 8, Table 1). The presence of lamellar formations like to the growth region is a characteristic feature for researched diamond crystals. Their appearance is usually caused by the inhomogeneous distribution of nitrogen or boron impurities during the crystal growth.

On the whole, the diamond crystals N1-N4 are polyblock, interfaces between blocks are mainly smallangle boundaries. Therefore, comparison of strains in the different local areas of crystals was interesting. As a result, on the basis of deformation diagrams (Fig. 8) and cathodoluminescent images (Figs 1a to 4a), it has been determined that the diamond crystal $\mathrm{N} 3$ is the most inhomogeneous, and the crystal N4 is the most homogeneous one.

Table 1. Kikuchi line width $L$ and relative changes of interplanar spacing $\Delta d / d$ for the crystal $N 4$.

\begin{tabular}{|c|c|c|c|c|}
\hline \multirow{2}{*}{$\begin{array}{c}\text { Crystal } \\
\text { area }\end{array}$} & \multicolumn{2}{|c|}{ Direction [100] } & \multicolumn{2}{c|}{ Direction [110] } \\
\cline { 2 - 5 } & $L$, pixels & $\Delta d / d, 10^{-4}$ & $L$, pixels & $\Delta d / d, 10^{-4}$ \\
\hline 1a & 39.578 & 0.278 & 23.443 & 1.334 \\
\hline 1b & 39.240 & 0.367 & 24.629 & 0.789 \\
\hline 1c & 39.446 & 0.313 & 24.614 & 0.795 \\
\hline 2a & 39.587 & 0.276 & 24.001 & 1.071 \\
\hline 2b & 39.410 & 0.322 & 24.695 & 0.760 \\
\hline 2c & 40.000 & 0.170 & 24.594 & 0.804 \\
\hline 3a & 40.613 & 0.016 & 25.140 & 0.569 \\
\hline 3c & 40.680 & 0.002 & 26.571 & 0.000 \\
\hline
\end{tabular}
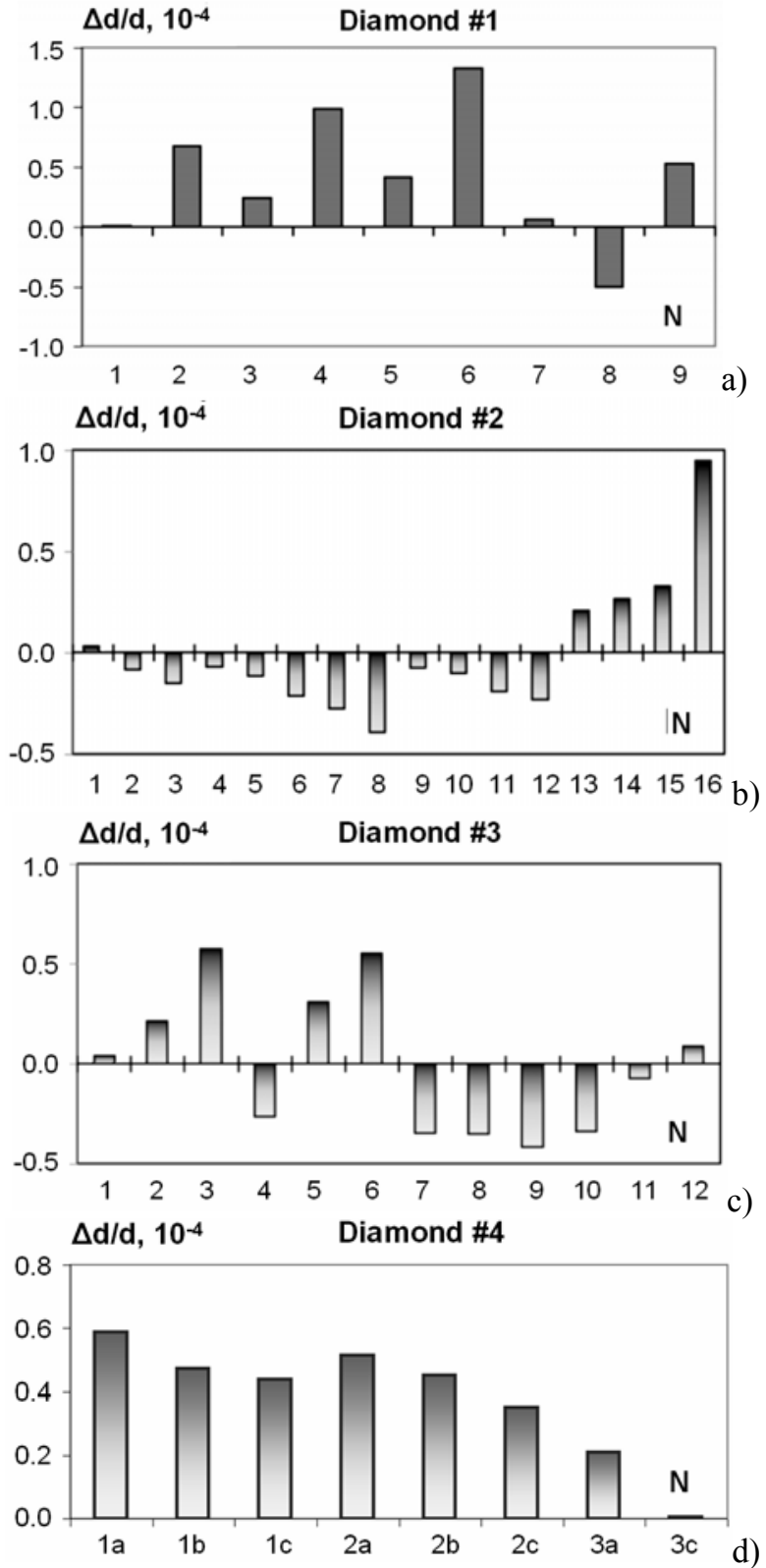

Fig. 8. Average values of $\Delta d / d$ determined for the diamond crystals N1-N4: a) sample N1; b) sample N2; c) sample N3; d) sample N4. Values of $\Delta d / d$ were determined relative to the area 1 for samples N1-N3, and relative to the area $3 \mathrm{c}$ for the sample N4.

\section{Analysis of Kikuchi pattern indicatrixes}

In the course of the analysis of Kikuchi line widths, the data processing for separate fragments of Kikuchi patterns was carry out, therefore it is necessary to select these fragments in the manual mode. For the software processing the Kikuchi patterns, the changes of Kikuchi line widths were determined using changes of pattern indicatrixes [19-21]. The indicatrixes of lines have been calculated on the basis of Fourier transform of Kikuchi patterns by using the software „Create_FFT” created in MatLab (Fig. 9). Application of Fourier transformation 
for Kikuchi pattern analysis is justified by the fact that the indicatrix of Fourier transform (angular spectrum) depends on the intensity and width of the Kikuchi lines in the pattern.
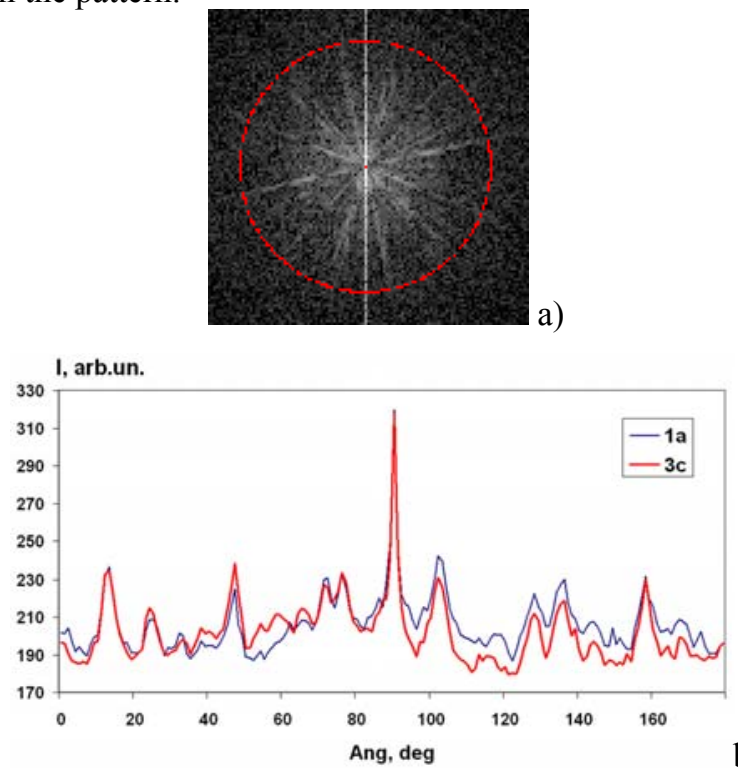

b)
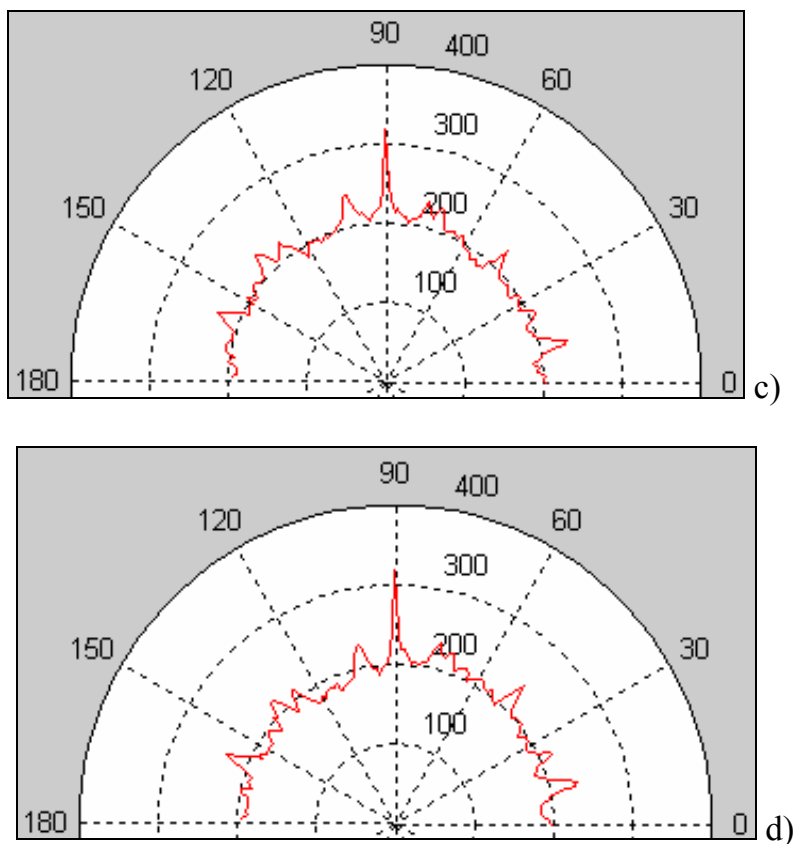

Fig. 9. Crystal N4: a) Image of Fourier transform of Kikuchi pattern obtained from the areas $1 \mathrm{a}$ and $3 \mathrm{c} ; \mathrm{b}$ ) comparison of indicatrixes for the areas $1 \mathrm{a}$ and $3 \mathrm{c} ; \mathrm{c}$ ) and $\mathrm{d}$ ) indicatrixes for the areas $1 \mathrm{a}$ and $3 \mathrm{c}$.

At the same time, the intensity and width of the Kikuchi lines depend on structural parameters of the researched crystal areas. Therefore, the changes of structural parameters for the corresponding areas can be defined using differences between indicatrix values. The calculation of Fourier transform indicatrix was performed by the software "Image_Indicatrix" (Delphi)

(Fig. 9). To obtain the indicatrix, the low frequency area of Fourier transform was used (Fig. 9a), because the spectrum of the Kikuchi lines predominates over the noise spectrum in this area.

$\Delta \mathrm{d} / \mathrm{d}, 10^{-4}$

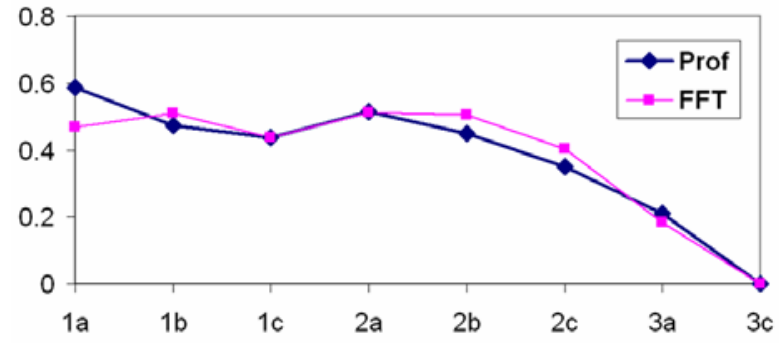

Fig. 10. Changes of $\Delta d / d$ values for the sample N4 relative to the area $3 \mathrm{c}$ determined with: profiles of intensity distribution of Kikuchi lines (Prof), and indicatrixes of Fourier transform of Kikuchi patternes (FFT).

Changes of interplanar spacing $\Delta d / d$ in the local areas relative to the area $3 \mathrm{c}$ were calculated on the basis of indicatrix differences for the crystal N4. Normalization of the values $\Delta d / d$ obtained from the indicatrix was performed to the $\Delta d / d$ values obtained on the basis of Kikuchi line profiles. Normalization was realized using the mean-square values of $\Delta d / d$ for all areas and complete agreement between $\Delta d / d$ values was obtained (Fig. 10).

\section{Conclusions}

1. The change in the intensity distribution of the Kikuchi line profiles allows to define the degree of structural homogeneity of local areas in synthetic diamond crystals, for example, to determine the local changes of interplanar spacing $\Delta d / d$.

2. Averaging-out the intensity distribution of the Kikuchi line profiles along certain directions allows to considerably decrease the casual constituent of intensity (noise) and increase information capability of the Kikuchi method.

3. Approximation of the intensity distribution in the Kikuchi line profiles by Gauss functions and developed software considerably increases exactness of determination of the line width and, accordingly, structural parameters of a crystal.

Thus, using electron diffraction (the Kikuchi method) allows to obtain information about mechanisms of forming the real crystal structure during the synthesis process, about dependence of diamond properties on its internal structure and degree of homogeneity.

\section{References}

2. P.R. Chalker, C. Johnston, M. Werner, Physical properties of diamond for thermistors and pressure 
transducers // Semicond. Sci. and Technol. 18, p. s113-s116 (2003).

2. J.M. Zuo, Quantitative convergent beam electron diffraction, Electron Crystallography. Determination of Nanosized Materials. Novel Approaches for Structure, Eds. T.E. Weirich, J.L. Labar, and X. Zou. Springer, Dordrecht, The Netherlands, Series II: Mathematics, Physics and Chemistry, 211, p. 143-168 (2006).

3. P. Schmid, M. Adamchik, E. Kohn, Design of high speed diamond microswitch // Semicond. Sci. and Technol. 18, p. s72-s76 (2003).

4. K. Thonke, The boron acceptor in diamond // Semicond. Sci. and Technol. 18, p. s20-s26 (2003).

5. C.E. Nebel, Electronic properties of CVD diamond // Semicond. Sci. and Technol. 18, p. s1-s11 (2003).

6. N.V. Novikov, Physical Properties of Diamond (Handbook), Published in Institute of Superhard Materials, p. 24-133 (1987).

7. T.D. Osetinskaya, V.N. Tkach, The effect of structure development in electron-irradiated type Ia diamond // J. Mater. Sci. 34, p. 2891-2897 (1999).

8. A.G. Gontar, V.N. Tkach, L.A. Romanko, Cathodoluminescent microtopography and spectroscopy of diamond produced by CVD and other methods // J. Chem. Vapor Deposition 4, p. 70-84 (1995).

9. A.S. Vishnevsky, N.N. Novikov, V.N. Tkach, Kosselegram using to determine the deformation of single crystals of synthetic diamond // Doklady Academii Nauk Ukrain. SSR, Ser. Fizika No.5, p. 55-59 (1984), in Russian.

10. K. Lonsdale, Divergent-beam X-ray photography of crystals // Phil. Trans. Roy. Soc. (London) 240(818), p. 219-250 (1947).

11. I.M. Fodchuk, M.D. Raransky, L.L. Gultay, O.O. Tkach, The features of anomalous multiple Xray transmission in Ge crystals with distorsions // J. Phys.D: Appl. Phys. 34, p. 93-97 (2001).

12. M.D. Borcha, S.V. Balovsyak, Y.D. Garabazhiv, V.M. Tkach, I.M. Fodchuk, Determination of the structural homogeneity of synthetic diamonds by Kikuchi diffraction method // Metallofizika $i$ noveishie tekhnologii, 31(9), p. 52-65 (2009).
13. I.M. Fodchuk, V.M. Tkach, V.G. Ralchenko, A.P. Bolshakov, E.E. Ashkinazi, I.I. Vlasov, Y.D. Garabazhiv, S.V. Balovsyak, S.V. Tkach, O.M. Kutsay, Distribution in angular mismatch between crystallites in diamond films // Diamond and Related Materials 19, p. 409-412 (2010).

14. E. Langer, R. Kurt, S. Dabritz, KOPSKO: a Computer Program for Generation of Kossel and Pseudo Kossel Diffraction Patterns // Cryst. Res. Technol. 34(7), p. 801-816 (1999).

15. R.P. Goehner and J.R. Michael, Phase identification in a scanning electron microscope using backscattered electron Kikuchi patterns // Journal of Research of the National Institute of Standards and Technology 101, p. 301-308 (1996).

16. H.A. Fowler and L. Marton, Widths of Kikuchi lines in germanium // J. Appl. Phys. 38, p. 1735 (1967).

17. B. Sur, R.B. Rogge, R.P. Hammond, V.N.P. Anghel, and J. Katsaras, Observation of Kossel and Kikuchi lines in thermal neutron incoherent scattering // Phys. Rev. Lett. 88(6), 065505-1 (2002).

18. L.S. Bushnev, Y.R. Kolobok, M.M. Myshlyaev, Fundamentals of Electron Microscopy. Publishing House of Tomsk State University, Tomsk, 1990 (in Russian).

19. B.V. Anisimov, V.D. Kurganov, V.K. Zlobin, Recognition and digital image processing, Vyshcha shkola, Kiev, 1983 (in Russian).

20. V.J. Kutkovetskyy, Pattern Recognition: Manual. Published in P. Mogyla Mykolaiv National University, Mykolaiv, 2003 (in Ukrainian).

21. H. Bubert, H. Jenett, Surface and Thin Film Analysis. Wiley-VCH Verlag, 2002. 